\title{
The concept of 'Translation': history and theory
}

\author{
IVAN A. KARPENKO
}

\begin{abstract}
This article deals with the problem of translations. It covers the history of translation in linguistics and analyzes peculiarities and role of translation in logic. Moreover, the article contains typical examples of embedding operations in terms of different logical theories.
\end{abstract}

Keywords: logic, translation, embedding, embedment, operation, language, calculi, theory

\section{1 'Theory of translation' in linguistics}

The French poet and philosopher, humanist Etienne Dolet [18, p. 6] in the XVI century was one of the first, who was trying to formulate a theory of translation in order to impart scientific justification into this kind of activity. According to his point of view, the true translation must meet the following criteria: perfect understanding of the content of the original text and the author's intentions; mastery of languages, which are involved in the translation process; inappropriateness of literal translation (in order to preserve the authentic atmosphere of the original); preserving the style of the original text, etc.

Later, another researcher T. Sevori [18, pp. 13-14] made a list of requirements for the various authors translations. Outlining of some of his statements will be enough to get his point of view on translation process: 1) the translation must convey the source words, 2) the translation must convey the source ideas, 3) the translation must be read like the original, 4) the translation must be read like a translation, etc. It is important to note that different scientists at different periods of time sometimes demanded from translation totally antithetic requirements. Hereafter, many translators, serious 
writers, and, finally, linguists made their own lists of requirements for 'translation' and then provide them with the corresponding theoretical justification. Here we are not going to discuss such research, carried out within this framework. Our main goal is to discuss the 'linguistic theory of translation', as so far scientific, well substantiated discipline.

The foundations of scientific translation theory were developed in the mid-twentieth century. That was the time of close attention of linguisticians and linguists to this problem. Some philosophers cast doubts on the possibility of translation in general - V. Humboldt, in particular [11]. According to him, every translation is an attempt to solve unsolvable task because of the dependence on the personal characteristics of the individual translator and his attitude to the text. Similar views served as the forerunners of the 'theory of untranslatability'.

The doubts on the possibility of the translation studies by the methods of linguistics were dispelled when this phenomenon became known as a special kind of verbal activity. This kind of activity was admitted to be the one, in which the units of the target language are selected depending on the specific language units of the source text, but not as a result of individual translation creativity. Here, apparently, there was a final demarcation of this area on the direct field of translation activities and the theory of translation.

J. Vinai and J. Darbelnet's attempt to subject different languages to the comparative analysis contributed a lot to the development of the linguistic theory of translation [29]. The analysis helps to detect units from different languages, which can be used in translation interchangeably. These words should a priori carry the same meanings in their two language systems or should come up as equivalents at the end of the translation.

Another linguist R. Jakobson [12] introduced an idea that the theory of translation plays an important role in other sciences, particularly in different branches of linguistics. He offered to define different types of translation: intralingual, interlingual and intersemiotic, in which one system of signs transforms into another. He supposed every transformation which carries the original meaning 
to be an adequate translation. This idea of R. Jakobson is closely correlated with the role of translation in logic.

G. Mounin in [21] paid special attention to the semantic structures of languages and claimed to square up to their differences. They occur because semantic discrepancies impose certain translation restrictions, for instance, make it impossible to render the original meaning fully.

It is also important to mention the merits of Russian scientists I.I. Revsin and V.U. Rosentsveig to the linguistic translation theory. In [26] they pointed out that the theory of translation should be not a prescriptive (i.e. the one that a priori formulates translation demands), but a descriptive - the one to describe the objective reality. It is the description that produces standards and regulatory guidelines of translation. They also highlighted the use of the deductive approach, which extends the use of general linguistic concepts in translation process.

I.I. Revsin and V.U. Rosentsveig defined two methods to transform source text to translated one: a) the direct substitution of the units of the source language into the units of another, b) interpretation. Last was meant to comprehend the reality at first, described by the original language, and then to descript it by the means of the target language.

J. Catford in [3] presumed that the central problem of the translation theory is to render the meaning of original and translated statements adequately close. In such a way he raised the question of texts equivalence in translation. He supposed that the original language meaning is replaced with the meaning of the target one, therefore the equivalence depends on the accuracy of such replacement.

V.N. Komissarov created an integrated theoretical conception in [17]. There he summarized different aspects of the linguistic analysis of translation, classified the research datum, including some mentioned above. Special attention was eftsoons paid to the problem of equivalence.

The issues stated above are just several fragments of the huge linguistic mosaics of translation theory problematic field. On the assumption of asserted, it is possible to point out two major infer- 
ences. These inferences will be the requirements basis for defining the term "translation" and for comparing other definitions with the worked out one later. First of all, any translation should keep the meaning of both the original and target texts. In other words, the problem of invariance should be taking into account while crossing the boundaries of two languages. Secondly, the target text should be theoretically equivalent to the original one. The logical sequence and inter-originating of these two principles can be clearly observed.

\section{2 'Translation' in logic}

The area of interest for this research includes particular use of translation method - more specifically, transfers between logical calculi. Hereunder, the concept of translation would require clarification. This concept stays in contrast to the linguistic requirements for translation, which state saving of semantic units as one of the key conditions. It is necessary to preserve the verity for compliance of translation in logic. That means, logical truths of one language should be translated into logical truths of another.

The consequence of the R. Yakobson's requirement about saving of semantic units is that 1) the true statement translation result is a true statement. As it was already mentioned, the logical verity is important in logic. Considering that, the adaptation in logic angle of the requirement 1) formulated above is the claim that 1) ${ }^{\prime}$ the true logic statement translation result is a true logic statement. Taking into consideration this requirement, the definition of translation will be:

Definition 1 (DF1). The translation of calculus $C_{1}$ into the calculus $C_{2}$ meant to be such a mapping of $\varphi$ set of all $L_{1}$-formulas of calculus $C_{1}$ into the set of all $L_{2}$-formulas of calculus $C_{2}$, that for every $L_{1}$-formula $A$ the following condition holds:

$$
\text { if } \vdash_{C_{1}} A \text {, then } \vdash_{C_{2}} \varphi(A) \text {. }
$$

However, any calculus will be transferred to any other calculus with this understanding of translation. That leads to questioning the retention of the source statement meaning - plus the fact, that logically true statements are not always equivalent in logical calculi. 
Besides, it remains unknown, how the translation result corresponds to the original. According to J. Catford, it is essential to estimate semantic affinity between the statements in the original and in the translation in order to verify their equivalence. In addition, this definition does not take into consideration the content of the statements. Therefore, it does not lead to source reconstitution by the translation, that is, in principle, impossible as it was indicated by G. Mounin.

Then let's introduce another requirement:

Definition 2 (DF2). The translation of calculus $C_{1}$ into the calculus $C_{2}$ meant to be such a mapping of $\varphi$ set of all $L_{1}$-formulas of calculus $C_{1}$ into the set of all $L_{2}$-formulas of calculus $C_{2}$, that for every $L_{1}$-formula $A$ the following condition holds:

$$
\vdash_{C_{1}} A \text {, if and only if } \vdash_{C_{2}} \varphi(A) \text {. }
$$

In contrast to the first definition, there is an opportunity to check the logical validity of the first calculus statement, considering the assumption of the logical validity of its image in the second calculus statement. Now it is possible to correlate the translation with the original. But most of the former lacks last even in this definition.

To avoid them, let's add to Df2 the following condition for avoiding those lacks $-\varphi$ is a recursive function. Then the new definition will be Df3.

But there are a number of drawbacks even in such way. According to R. Epstein [6, p. 291], for example, this definition does not guarantee the safety of the produced formulas structures. That means the impossibility of full source content reproduction on free analogy with Mounin's remark. Then it is necessary to take into account the structure of the formulas, that, in fact, is the requirement of inductive definition? (let's name this definition Df4).

Is it enough to give a good definition of the translation? Apparently, not. The question remains, whether the equivalence of the original and the translation result observes?

It is necessary to work out more strict criteria for what is called 'translation'. Below such definitions proposed directly by logics will be considered. 


\section{Definitions of embedding operations}

Let's analyze and compare the most important, in our opinion, definitions, suggested by V.A. Smirnov, R. Wojcicki and R. Epstein.

In [28] V.A. Smirnov defined the translations between theories. However, his definition will also be relevant for the calculi with the appropriate modification.

Suppose, that $T_{1}$ and $T_{2}$ are theories, formulated accordingly in languages $L_{1}$ and $L_{2}$ with the corresponding logics. Suppose, that $\varphi$ is a recursive function that matches formulas in language $L_{1}$ with formulas in language $L_{2}$ for any $L_{1}$-formula $A$. The function meant to be called translation of theory $T_{1}$ to $T_{2}$, if the following condition holds: if $A \in \mathrm{T}_{1}$, then $\varphi(\mathrm{A}) \in \mathrm{T}_{2}$. If the following additional condition holds: if $\varphi(\mathrm{A}) \in \mathrm{T}_{2}$, then $A \in \mathrm{T}_{1}$, then the recursive function $\varphi$ would be called an embedding operation of the theory $T_{1}$ to the theory $T_{2}$. Theory $T_{1}$ could be embedded to the theory $T_{2}$, if and only if there is a recursive function, which embeds $T_{1}$ to $T_{2}$. Of course, if there is a translation - there is not necessarily the case of embedment. Later we will talk more about the 'embedment' since we are deeply interested in cases that satisfy both Smirnov's conditions at once.

V. A. Smirnov's definition actually coincides with the above Df3.

R. Wòjcicki offered a different definition [30].

Mapping $\varphi$ from sentential language $L_{1}$ to sentential language $L_{2}$, which have the same set of propositional variables, is called the embedment if and only if the following two conditions hold:

1. There is a formula $\phi\left(p_{0}\right)$ from one propositional variable $p_{0}$ in $L_{2}$, such that for every propositional variable $p, \varphi(p)=\phi\left(p_{0}\right)$.

2. For each logical connective $r_{i}$ in $L_{1}$ there is a formula $\phi_{i}$ in $L_{2}$, such that for all $\alpha_{1}, \ldots, \alpha_{k}$ in $L_{1}, k$ is the arity $r_{i}$,

$$
\varphi\left(r_{i}\left(\alpha_{1}, \ldots, \alpha_{k}\right)\right)=\phi_{i}\left(p_{1} / \varphi\left(\alpha_{1}\right), \ldots, p_{k} / \varphi\left(\alpha_{k}\right)\right) .
$$

Then, the definition of embedment is stated for propositional calculi and theories.

Suppose that $C_{1}=\left(L_{1}, C_{1}\right), C_{2}=\left(L_{2}, C_{2}\right)$ are some propositional calculi, and $T_{1}=\left(L_{1}, T_{1}\right), T_{2}=\left(L_{2}, X_{2}\right)$ are some theories, 
then $\varphi$ is the embedding operation from $L_{1}$ to $L_{2}$, if for every $T \subseteq L_{1}$ and for every $\alpha \in L_{1}$ the following holds:

$$
\alpha \in \mathrm{C}_{1}(T) \text { if and only if } \varphi(\alpha) \in \mathrm{C}_{2}(\varphi(T))\left(\varphi\left(T_{1}\right)=T_{2}\right) .
$$

Epstein offered a similar definition (ref. [9, pp. 290-291]), but with some differences, which will be defined in detail below.

At first, he formulated the definition of a mapping from one logic to another. This helps to preserve the relation of deductivity.

Mapping of the propositional logic $L$ to the propositional logic $M$, which preserves the relation of deductivity, is a mapping $\varphi$ from language $L_{L}$ to language $L_{M}$ ( $L_{L}$ и $L_{M}$ are the languages of logics $L$ and $M$, respectively), such that for every formula $A$ the following condition holds:

$$
\vdash_{L} A \text { if and only if } \vdash_{M} \varphi(A) \text {. }
$$

Mapping is a translation, if for any $\mathrm{G}$ and $\mathrm{A}$ the following condition holds:

$$
\begin{gathered}
G \vdash_{L} A \text { if and only if } \varphi(G) \vdash_{M} \varphi(A), \text { where } G \text { is the set of } \\
\text { formulas, and } \varphi(G)=\{\varphi(A): A \in G\} .
\end{gathered}
$$

According to Epstein, such definition of embedment does not cause the preservation of target language structure. This appears to be valid, especially while discussing such examples, as Glivenko's translation of classical propositional logic into the intuitionistic logic [9], which matches the above specified definition.

Epstein then introduced the concept of grammatical mapping:

Mapping $\varphi$ of propositional language $L_{1 \supset \neg}$ to any propositional language $L_{2}$ is called grammatical, if there are schemes $\lambda, \varphi, \psi$ in language $L_{2}$, such that

$$
\begin{gathered}
p^{*}=\lambda(p), \\
(\neg A)^{*}=\varphi\left(A^{*}\right), \\
(A \supset B)^{*}=\psi\left(A^{*}, B^{*}\right),
\end{gathered}
$$

whereas it is implied, that in these languages, the set of propositional variables is the same. 
Grammatical mapping is called homophonic if each connective maps into itself. Grammatical embedment is a grammatical mapping, which comes out as an embedment.

R. Wòjcicki and R. Epstein's definitions meet the one, which was marked above as Df4.

\section{Comparison of definitions}

As is clear from aforementioned definitions, all of them have specific differences. V.A. Smirnov provides the broadest definition. Glivenko's contribution resulted in translation of the classical propositional logic into the intuitionistic one. His translation meets the definition of V. A. Smirnov, but falls outside the scope of R. Wòjcicki and R. Epstein's meaning of embedment.

Let us denote the differences in the definitions of Wòjcicki and Epstein. There is a need to reformulate them in a similar style and then compare.

Suppose the languages $L^{\prime}$ and $L^{\prime \prime}$ are given with one and the same set of propositional variables, $\supset$ and $\neg$ are the logical connectives of $L^{\prime}$ language, $\varphi$ is the embedding operation from $L^{\prime}$ language to $L^{\prime \prime}$, and $\boldsymbol{A}, \boldsymbol{B}, \boldsymbol{C}$ are some formulas of $L^{\prime \prime}$ language.

Then the definition of Wòjcicki can be represented in the following form:

- $\varphi(p)=\left[p_{0} / p\right] \boldsymbol{A}$, where $\left[p_{0} / p\right] \boldsymbol{A}$ is the result of substitution $p$ instead of $p_{0}$ into the formula $\boldsymbol{A}$, which does not contain occurrences of propositional variables, other than $p_{0}$,

- $\varphi(A \supset B)=\left[p_{1} / \varphi(A), p_{2} / \varphi(B)\right] B$, where $\left[p_{1} / \varphi(A)\right.$, $\left.p_{2} / \varphi(B)\right] \boldsymbol{B}$ is the result of substitution $\varphi(A)$ instead of $p_{1}$ and $\varphi(B)$ instead of $p_{2}$ into the formula $\boldsymbol{B}$,

- $\varphi(\neg A)=\left[p_{1} / \varphi(A)\right] \boldsymbol{C}$, where $\left[p_{1} / \varphi(A)\right] \boldsymbol{C}$ is the result of substitution $\varphi(A)$ instead of $p_{1}$ into the formula $C$, which does not contain occurrences of propositional variables, other than $p_{1}$. 
Epstein claims the following:

- $\varphi(p)=\left[p_{0} / p\right] \boldsymbol{A}$, where $\left[p_{0} / p\right] \boldsymbol{A}$ is the result of the substitution $p$ instead of $p_{0}$ into the formula $\boldsymbol{A}$,

- $\varphi(A \quad \supset B)=\left[p_{1} / \varphi(A), p_{2} / \varphi(B)\right] \boldsymbol{B}$, where $\left[p_{1} / \varphi(A)\right.$, $\left.p_{2} / \varphi(B)\right] \boldsymbol{B}$ is the result of substitution $\varphi(A)$ instead of $p_{1}$ into the formula $\boldsymbol{B}$,

- $\varphi(\neg A)=\left[p_{1} / \varphi(A)\right] C$, where $\left[p_{1} / \varphi(A)\right] C$ is the result of substitution $\varphi(A)$ instead of $p_{1}$ into the formula $C$, which does not contain occurrences of propositional variables, other than $p_{1}$.

So the only varying cases are those, which hold the scope of the embedding operation within propositional variables. Otherwise, $\mathrm{R}$. Wòjcicki and P. Epstein's definitions stay matching.

But wherein does the complexity of embedment universal definition as a means for comparison and study of logical systems lie? As referred to M.N. Rybakov and A.V. Chagrov in [4], it makes sense to impose additional conditions (other than those, that were put forward in the above definition), depending on the purpose of a particular embedment, since it is often necessary to consider the contents of the formulas. Otherwise, it is impossible to represent adequately the formulas of one logic by the means of another logic. In other words, in any embedment could be a list of requirements for embedding operation.

The definition proposed by V.A. Smirnov would be the basis for this research on different embedments of logical calculi. This was motivated by simplicity and convenience of his definition. But our embedding operations are also true within R. Wòjcicki and R. Epstein's theories, as it will become clear from their construction.

\section{Reasons for embedding operations application in logic. Philosophical and technical aspects}

There are several reasons for the use of embedding operations in logic. Here we can highlight technical and philosophical aspects.

As it was mentioned above, philosophically, embedment helps to map one theory by another theory terms. The ability of comparing 
theories, formulated in different languages, becomes real with such a phenomena, but stands in opposition to Firebrand's idea of 'incommensurability of theories'. According to this idea terms of one theory cannot be expressed in terms of another one, as they themselves have different meanings. The embedding operations method (if we can call it a method) removes the 'problem of understanding'. If we embed non-interpreted calculus into calculus, which has some semantic meaning, the interpretation of first embedment formulas becomes possible.

This raises the problem of negation in language: embedment of containing negation language to a different positive language or its own positive part we get an opportunity to speak about the first language facts using only affirmative sentences, i.e. without saying any 'no'.

Speaking about the technical aspect we should note the problem of decidability. Hereby, embedment of any calculus into a decidable one results into solving the problem of decidability of the first calculus. The problem of languages relative insolvability is also observed in terms of this problem. Embedment of calculi into their own fragments and other calculi fragments decreases the number of connectives, which are necessary to express formulas in different languages. This is again very important for understanding of the original calculi. V.M. Popov got one of the most eloquent and unexpected results in this area, when he embedded classical propositional logic into its implicative fragment and implicative fragment of intuitionistic propositional logic. We think that this idea demands some serious deliberation, as it is not quite clear.

Besides, as it was shown in V.M. Popov's research in [22], use of the embedding operations helps to prove fragment severability in calculi. The characteristics, mentioned here, can be added to much broader list of application areas than discussed here.

\section{The history of the concept of 'embedment'. Specific embedding operations in classical and intuitionistic propositional logics}

Besides the above mentioned Smirnov, Wòjcicki and Epstein, attempts to determine and organize embedding operations were also 
taken by N.A. Shanin [27], who, according to V.A. Smirnov (ref. [28, p. 120]), had first coined the term, D. Prawitz and P. Mamnos [25], W.A. Carnielli and M.L. D'Ottaviano [2]. Based upon the last work of Carnielli and D'Ottaviano, A.S. Karpenko concluded that the application of embedding operations is a key tendency in the development of contemporary logic [13].

The term of embedding operation was first time introduced, according to V.A. Smirnov [28, p. 120], by A. N. Kolmogorov in 1925 [16] while embedding classical logic into intuitionistic. Particular attention is worth paying to the problem of relation between classical and intuitionistic logics towards the embedding operations after many leading scientists who made it a point.

For that let's specify after [15] the calculi $P C$ (classical propositional calculus) and Int (intuitionistic propositional calculus).

Language $L_{\wedge \vee \supset \neg}$ of these calculi is the conventionally determined propositional language with a set of propositional variables $\left\{p_{1}, p_{2}, p_{3}, \ldots\right\}$.

Calculi $P C$ and Int are the calculi of Hilbert type with the conventionally determined concept of proof. The set of deduction rules for each of these calculi has the only rule: A, A $\supset$ B/B. Therefore, it is sufficient to define the set of its axioms in order to specify any of these calculi.

Calculus $P C$. The set of all axioms of calculus $P C$ is the set of all formulas, each of which is stated in at least one of the following types:

1. $A \supset(B \supset A)$,

2. $A \supset(B \supset C) \supset((A \supset B) \supset(A \supset C))$,

3. $(A \wedge B) \supset A$,

4. $(A \wedge B) \supset B$,

5. $A \supset(B \supset(A \wedge B))$,

6. $A \supset(A \vee B)$,

7. $B \supset(A \vee B)$, 
8. $(A \supset C) \supset((B \supset C) \supset((A \vee B) \supset C))$,

9. $(A \supset B) \supset((A \supset(\neg B)) \supset(\neg A))$,

10. $(\neg(\neg A)) \supset A$.

Calculus Int. The set of all axioms of Int is the union of the set of all formulas, each of which is a formula of at least one of the above mentioned 1-8 types with the set of all formulas of the type:

$9^{\prime}(\neg A) \supset(A \supset B)$.

Glivenko in 1929 suggested embedding operation for classical and intuitionistic logics, which lies within the language $L-$ the conventionally determined propositional language with a set of logical connectives $\{\wedge, \vee, \supset, \neg\}$. This embedding operation associates each $L$-formula $A$ with $L$-formula $\neg(\neg A)$. Precisely, Glivenko proved the following theorem, which let's call T1 for convenience of reference.

Theorem 1 (T1). $G \vdash_{P C} A$ if and only if $\neg(\neg G) \vdash_{P C} \neg(\neg A)$.

V. Popov noted an interesting fact about Glivenko's embedding operation to fail for the predicative versions of intuitionistic and classical logics.

In 1933 Gödel showed [10] that Int could be considered as the extension of classical propositional logic, formulated in the language $L_{\wedge\urcorner}$ within the meaning of the following theorem (let's call it T2).

Theorem 2 (T2). For $L_{\wedge \neg}$-formula $A$ it is true that $\vdash_{P C_{\wedge \neg}} A$ if and only if $\vdash_{I N T} A$.

Proof. The proof of this theorem right to left is obvious, because the set of all Int theorems is included into the set of all $P C$ theorems. Let's prove that if $\vdash_{P C_{\wedge}} A$, then $\vdash_{I N T} A$.

The proof is carried out by induction on the structure of $L_{\wedge\urcorner^{-}}$ formula $A$.

Now there are three options: 1) $A$ is a propositional variable $p_{i}$, 2) $\mathrm{A}$ is $\neg \mathrm{B}$, 3) $A$ is $\mathrm{B}_{1} \wedge \mathrm{B}_{2}$.

Let's consider 1). Here the following statement requires provement - if $\vdash_{\left.P C_{\wedge}\right\urcorner} p_{i}$, then $\vdash_{I N T} p_{i}$. But none of propositional variables can be the theorem of $P C$ calculi. Therefore, the theorem 
would be true here, taking into consideration the characteristics of the classical implication.

Let's consider 2). Here the following statement requires provement - if $\vdash_{\left.P C_{\wedge}\right\urcorner} \neg B$, then $\vdash_{I N T} \neg B$. But after Glivenko's result [9] that $\vdash_{P C} \neg A$ if and only if $\vdash_{I N T} \neg A$ the previous statement would be true.

Let's consider 3). Here the following statement requires provement - if $\vdash_{P C_{\wedge} \rightarrow} \mathrm{B}_{1} \wedge \mathrm{B}_{2}$, then $\vdash_{I N T} \mathrm{~B}_{1} \wedge \mathrm{B}_{2}$. Taking into consideration the characteristics of $P C_{\wedge\urcorner}$ it is true that $\left.a\right)$ if $\vdash_{P C_{\wedge\urcorner}} \mathrm{B}_{1} \wedge \mathrm{B}_{2}$, then $\vdash_{P C_{\wedge\urcorner}} \mathrm{B}_{1}$ and $\vdash_{P C_{\wedge}} \mathrm{B}_{2}$.

Using the inductive assumption, we have: b) if $\vdash_{P C_{\wedge}} B_{1}$, then $\vdash_{I N T} B_{1}$ and $\vdash_{P C_{\wedge}} B_{2}$, then $\vdash_{I N T} B_{2}$.

In Int the following formula is provable: $c) \mathrm{B}_{1} \supset\left(\mathrm{B}_{2} \supset\left(\mathrm{B}_{1} \wedge \mathrm{B}_{2}\right)\right)$. From $a), b)$ and $c$ ) by the definition of Int proof, we obtain that $\vdash_{I N T} \mathrm{~B}_{1} \wedge \mathrm{B}_{2}$.

Thus, the theorem T2 is proved.

Now let's introduce the result of Łukasiewicz [19] on embedment of $P C$ into Int, when the binary identical relations of classical logic were used for connectives $\supset$ and $\vee$. This was initiated for representing the $P C$ formulas, stated in the $L_{\wedge \vee \supset \neg}$ language, into the Int formulas. There has been constructed the following embedding operation (here and below we will use the original symbols for embedding operations):

- $p^{*}=p$

- $(A \wedge B)^{*}=A^{*} \wedge B^{*}$

- $(\neg A)^{*}=\neg\left(A^{*}\right)$

- $(A \vee B)^{*}=\neg\left(\left(\neg A^{*}\right) \wedge\left(\neg B^{*}\right)\right)$

- $(A \supset B)^{*}=\neg\left(A^{*} \wedge\left(\neg B^{*}\right)\right)$.

Theorem $3(\mathrm{~T} 3) . \vdash_{P C} A$ if and only if $\vdash_{I N T} A^{*}$.

According to Epstein (ref. [6, p. 213]), neither the embedment in the sense of $\mathrm{T} 2$, nor the embedment in the sense of $\mathrm{T} 3$ preserves the relations of consequences. That happens because these theorems 
are false in the wording of $G \vdash_{P C} A$ if and only if $G \vdash_{I N T} A$. For example, if we have $\neg \neg p \vdash_{P C} p$, then we also have $\neg \neg p \vdash_{I N T} p$, and hence we obtain by the theorem of deduction the false statement $\vdash_{I N T} \neg \neg p \supset p$.

Proposed by Gentzen in 1936 [8], his embedment preserves the relation of consequences. This statement consists of the language $L(\neg, \supset, \wedge, \vee)$ and the following embedding operation:

- $p^{\circ}=\neg \neg p$

- $(A \wedge B)^{\circ}=A^{\circ} \wedge B^{\circ}$

- $(\neg A)^{\circ}=\neg\left(A^{\circ}\right)$

- $(A \vee B)^{\circ}=\neg\left(\left(\neg A^{\circ}\right) \wedge\left(\neg B^{\circ}\right)\right)$

- $(A \supset B)^{\circ}=A^{\circ} \supset B^{\circ}$,

Theorem 4 (T4). $G \vdash_{P C} A$ if and only if $G^{\circ} \vdash_{I N T} A^{\circ}$.

Here Gentzen managed to preserve the relation of consequences while embedment process exactly because of non-standard mapping of propositional variable (through double negation).

It also makes sense to specify here the aforementioned significant result of V.M. Popov [23] in order to close the review of the history of embedment of classical propositional logic into intuitionistic.

Classical propositional logic is axiomatized by calculus $C l_{\supset f}$. Its axioms are those and only those $L_{\supset}$-formulas, each of which is given as $A \supset(B \supset A)$ or $(A \supset B) \supset((A \supset(B \supset C)) \supset(A \supset C))$ or $((A \supset f) \supset f) \supset A$. The inference rule here: $A, A \supset B / B$.

Implicative fragment of intuitionistic propositional logic is axiomatized by calculus Int $_{\supset}$. Its axioms are those and only those $L_{\supset}$-formulas, each of which is given as $A \supset(B \supset A)$ or $(A \supset B) \supset$ $((A \supset(B \supset C)) \supset(A \supset C))$. The inference rule here: $A, A \supset B / B$.

The following operations are offered here: $S d$ (first introduced by V.M. Popov in [24]), and T. Sd is meant to be a mapping of the set of all $L_{\supset}$-formulas into the set of all $L_{\supset}$-formulas, and $T$ is a mapping of the set of all $L_{\supset}$-formulas into the set of all $L_{\supset}$-formulas. 
- $S d(f)=p_{1}$,

- $S d\left(p_{i}\right)=p_{i+1}($ where $i \in\{1,2,3, \ldots\})$,

- $S d(A \supset B)=S d(A) \supset S d(B)$.

- $\mathrm{T}\left(p_{1}\right)=p_{1}$,

- $T\left(p_{i}\right)=\left(p_{i} \supset p_{1}\right) \supset p_{1}$ (where $\left.i \in\{1,2,3, \ldots\}\right)$,

- $T(A \supset B)=T(A) \supset T(B)$.

Further the theorem provement takes place (T5 in our notation). TheOREM $5(\mathrm{~T} 5) . \vdash_{S d \supset f} \mathrm{~A}$ if and only if $\vdash_{\text {Int }} \mathrm{T}(\operatorname{Sd}(\mathrm{A}))$.

V.A. Bocharov, M. Zaharyashev, V.I. Markin, A.V. Chagrov, L.L. Esakia should be also mentioned as the contemporary Russian scientists who gave their tribute to the study of logical systems through embedding operations. V.A. Bocharov in [1] constructs embedment of Boolean algebra into syllogistics, among other works on this subject. V.I. Markin in his book [20, pp. 35-43] embedded the systems of clear positive Aristotelian syllogistics into the predicate calculus. A translation from the calculus $R M$ to the positive fragment of $R M$ is constructed in [14]. Work of M. Zaharyashev and A.V. Chagrov [5] is dedicated to embedment of intuitionistic logic and its extensions to different normal modal logics. L.L. Esakia in [7] considers new aspects of Gödel's embedment of intuitionistic logic into modal logic $S_{4}$.

\section{References}

[1] Bocharov, V. A., Boolean algebra in the terms of syllogistics, in V. A. Smirnov (ed.), Proceedings of the Research Logical Seminar of Institute of Philosophy of Russian Academy of Sciences, IFRAN, Moscow, 1983, pp. 32-42 (in Russian).

[2] Carnielli, W. A., and M. L. D'Ottaviano, Translations between logical systems: A MANIFESTO, Logique et Analyse 157:67-81, 1997.

[3] Catford, J. C., A Linguistic Theory of Translation: An Essay in Applied Linguistics, Oxford University Press, London, 1965.

[4] Chagrov, A. V., and M. N. Rybakov, Standard translations of nonclassical formulae and relative decidability of logics, in A. S. Karpenko 
(ed.), Proceedings of the Research Logical Seminar of Institute of Philosophy of Russian Academy of Sciences. IFRAN, Moscow, 2000, pp. 81-98 (in Russian).

[5] Chagrov, A., and M. Zakharyashchev, Modal Companions of Intermediate Propositional Logics, Studia Logica 51(1):49-82, 1992.

[6] Epstein, R. L., The Semantic Foundations of Logic. Vol.1: Propositional Logic, Kluwer, Dordrecht, 1990.

[7] Esakia, L., Gödel embedding of the intuitionistic calculus to modal logic: recent and new observations, in A. S. Karpenko (ed.), Smirnov Readings. 4th International Conference, IFRAN, Moscow, 2003, pp. 7275 .

[8] Gentzen, G., Die Widerspruchsfreiheit der reinen Zahlentheorie. Mathematische Annalen 112:493--565, 1936.

[9] Glivenko, M., Sur quelques points de la logique de M. Brouwer, Acadèmie Royale de Belgique, Bulletins de la classe des sciences 5(15):183-188, 1929.

[10] GöDEL, K., On intuitionistic arithmetic and number theory, in K. Gödel, Collected Works, Vol. 1, Oxford University Press, New York, 1986, pp. 287-295.

[11] Von Humboldt, W., Sein Leben und Wirken dargestellt in Briefen, Tagebuchern und Dokumenten seiner Zeit, Berlin, 1953.

[12] Jakobson, R., On linguistic aspects of translations, in R. Brower (ed.), On Translation, OUP, New York, 1959, pp. 232-239.

[13] Karpenko, A. S., Logic on the boundary of millennia, Logical Investigations 7:7-60, 2000 (in Russian).

[14] Karpenko, I. A., and V. M. Popov, Embedding of calculus RM into its positive fragment, Logical Investigations 10:94-99, 2001 (in Russian).

[15] Kleene, S. C., Introduction to Metamathematics, D. Van Nostrand Company, New York, 1952.

[16] Kolmogorov, A. N, On the tertium non datur principle, Matematicheskij sbornik 32(4):646-667, 1925 (in Russian).

[17] Komissarov, V. N., Linguistics of Translation, Mezhdunarodnye otnosheniya, Moscow, 1980 (in Russian).

[18] Komissarov, V. N., Theory of Translation (linguistic aspects), Vysshaya shkola, Moscow, 1990 (in Russian).

[19] EukAsiewicz, J., On the intuitionistic theory of deduction, Indagationes Mathematicae 14:202-212, 1952. 
[20] Markin, V. I., Syllogistic theories in modern logic, Moscow State University, Moscow, 1991 (in Russian).

[21] Mounin, G., Les problems théoriques de la traduction, Paris, 1963.

[22] Popov, V. M., Embedding of intuitionistic propositional calculus into its positive fragment, Logical Investigations, 8:150-154, 2001(in Russian).

[23] Popov, V. M., Embedment of classical propositional logic into its implicative fragment and into implicative fragment of intuitionistic propositional logic, in A. S. Karpenko (ed.), Proceedings of the Research Logical Seminar of Institute of Philosophy Russian Academy of Sciences, IFRAN, Moscow, 2000, pp. 36-41 (in Russian).

[24] Popov, V. M., Embedment of implicative fragment of classical propositional logic into implicative fragment of intuitionistic logic, Logical Investigations 7:80-83, 2000 (in Russian).

[25] Prawitz, D., and P. E. Malmnäs, A survey of some connections between classical, intuitionistic and minimal logic, in H. A. Schmidt et al. (eds.), Contributions to Mathematical Logic, North-Holland, Amsterdam, 1968, pp. 215-229.

[26] Revzin, I. I., and V. U. Rozentsveig, Basics of General and Machine Translation, Vysshaya shkola, Moscow, 1964 (in Russian).

[27] Shanin, N. A., On some logical problems of arithmetic, Studies of $V$. A. Steklov Mathematical Institute 43:3-112, 1955 (in Russian).

[28] Smirnov, V. A., Logical Methods of Scientific Knowledge Analysis, URSS, Moscow, 2002, 2nd ed. (in Russian).

[29] Vinai, J. P., et J. DARBelnet, Stylistique contractee du français et de l'anglais, Paris, 1958.

[30] WÒjcicki, R., Theory of Logical Calculi: Basic Theory of Consequence Operations, Dordrecht, Kluwer, 1988. 